\title{
Directing Status Messages to their Audience in Online Communities
}

\author{
Milan Stankovic, Philippe Laublet \\ LaLIC \\ Université Paris IV - Sorbonne, Paris, France \\ milan@milstan.net, philippe.laublet@paris-sorbonne.fr
}

\author{
Alexandre Passant \\ Digital Enterprise Research Institute \\ NUI-Galway, Galway, Ireland \\ alexandre.passant@deri.org
}

\begin{abstract}
In this paper we present the results of our user study about status message sharing on the Social Web. The study revealed the privacy and information noise (sometimes originating from gap of understanding and sometimes from lack of significance) to be the key problems in the domain and allowed us to unveil their nature. Further on we present the existing solutions and workarounds for those problems and introduce the idea that Semantic Web technologies could help confront those problems in a more complete way. We propose a way to use semantic descriptions of status messages, their intended audiences and distributed data about users to direct status messages to their intended recipients. Particularly, we rely on the Online Presence Ontology as a vocabulary for exposing status message semantics, and we provide necessary extensions to support status message directing.
\end{abstract}

Index Terms-Faceted Identity, Linked Data, Online Presence, Social Web, Social Networks.

\section{INTRODUCTION}

S TATUS messages are short textual expressions that describe the state of a user's presence in the online world. Sharing status messages on different social services on the Web (Microblogging services, Instant Messaging platforms, Social Networks) became a common practice for people to share thoughts, feelings of the moment, announce one's presence in the online world and broadcast information. However, as more and more users take part in status message sharing, the open communities become overloaded with status updates. Many problems arise from such an overload. Firstly, confidentiality of status messages in open communities is a significant question, since not all status messages are meant for general public. Some should be kept private from certain contacts who might use them in an inappropriate way. An example could be a status message revealing somebody's drinking habits, meant to amuse personal friends, but the same status message could be a source of inconvenience if shown to work colleagues.

Apart from private nature of some status messages there are other reasons why a particular status message might not be

Manuscript received July 30, 2009.

The work presented in this paper has been funded in part by Science Foundation Ireland under Grant No. SFI/08/CE/I1380 (Lìn-2). suitable for a certain audience. For example, some status message updates may have no significance for certain groups of contacts that consider them as information noise. It is a common case that we subscribe to someone's statuses because of the interest in professional news he/she is sharing, but aside we get a lot of postings about the person's personal life that don't interest us. Problems like those limit in a great deal, the usefulness of today's status sharing services (mostly microblogging services and Social Networks)

In order to explore more deeply the nature of the problem of confidentiality and other key problems in status message sharing in large communities on the Social Web we conducted a qualitative user study with subjects who are using status messages for different purposes and in different contexts. The goal of the study was to develop understanding of the key problems, factors that make a status message open or confidential - that determine its intended audience. Apart from understanding the problems, the study allowed us to explore the space of possible solutions. In Section 2 we present the results of our user study. Section 3 presents the Presence Diamond, a useful notion for the study of presence online as a faceted phenomenon. Section 4 lists currently available solutions for problems identified in the study. In Section 5 we introduce a way to direct a status message to its intended audience using Semantic Web technologies, and we show how those technologies are flexible to support even dynamic audience definitions (where members of the audience change frequently). Section 6 presents related work and in Section 7 we conclude the paper.

\section{THE USER STUDY}

The user study was conducted through a series of ten interviews with users of social networks and microblogging platforms who have been using them for status message sharing for some time (a year in average). The 30-35 minute interviews were field-noted and audio recorded for further reference. Users' age ranged from 22 to 35 . This choice proved to correspond well to demographics of users of the most active microblogging services (documented in a statistical report done by Pew Internet ${ }^{1}$ ). Equal number of male and female subjects, from France and Ireland, with different

${ }^{1}$ http://www.pewinternet.org/Reports/2009/Twitter-and-status-updating.aspx 
origins and backgrounds, took part in the interviews.

After a couple of questions about users' background, users were asked to tell their status message publishing experiences. The main goal was to identify their context in the time of publishing, nature of the status message content and the intended audience. The inconveniences and the inability of microblogging tools and social networks to meet their status message sharing needs were also explored.

Once we collected the user stories, we relied on Grounded Theory inspired approach to extract relevant categories from them, and further generalize the categories to super-categories that we call - major issues. Open and Axial coding were used with participation of two researchers in order to reduce the impact of subjectivity.

Grounded Theory was introduced by Glaser and Strauss [1] and has served ever since for analysis of results in qualitative research in Social Sciences. Grounded Theory is an approach to looking systematically at qualitative data to derive codes and group them into relevant categories that will further be generalized into concepts that make the ground for generating a theory. Generalizations are derived by thinking efforts of researchers. Due to a space limit, in this paper we present only a part of our findings - the highest level generalizations, and we briefly describe them with some of the lower level generalizations that we find the most relevant to our intended readers.

Generally we discovered that many times when users publish a status message, they have a certain audience in mind. The status message is intended for a particular audience either because of its ability to understand the message (or the inability of others to understand it properly) either because of significance of the message for a certain group (and insignificance for others) or because of the confidential nature of the status message content. The next three sections present those major issues - reasons why a status message has its particular audience.

\section{A. Gap of Understanding}

In many cases where a certain status message is not meant for a certain group of people it is because of their inability to understand, properly interpret and maybe even reply to the content of the message. Sometimes the inability arises from shallow acquaintance like in cases where the user publishing a status messages knows a certain group of people for a short time. The shallowness of acquaintance can be an obstacle for this group of people to understand jokes, metaphors and properly interpret the intended meanings of status messages. Sometimes the gap of understanding results from lack of competence like in cases where users use status messages to ask for advice, or provoke professional discussions. This problem is also present in scenarios of automatic postings of status messages across services (e.g. automatic forwarding from Twitter to Facebook) where mostly different audiences are present on different services. Quite often personal friends from one service (Facebook in our case) don't understand and find irrelevant the profession-related status messages posted on another service (Twitter in our case).

Some status messages bear a socially established meaning, understood by a small community of people, like those containing internal jokes, or internal aliases and metaphors. Such status messages may be misinterpreted by people outside that small community and may be source of misunderstandings, inappropriate comments and other inconveniences.

\section{B. Lack of Significance}

In other cases, a status message is not intended for some people simply because they have no interest in it. This is the case when a status message relates to a certain domain and thus can be of significance only to people with an interest in the domain. This case is common when people make connections based on a shared interest, stay in touch and then use status messages to spread domain related news, announce events and provoke discussions. In some cases it is the interest in the domain that makes a certain group of people not interested in other non domain-related status messages of a user. For people who are not familiar with the domain such messages can represent noise.

In other cases some groups of people might not be able to make use of the information in the status message which has an informative purpose. This is the case with status messages highly dependent on location - like those containing invitations to local parties and announcements of local events. In both cases such status messages are irrelevant to people from other locations who could not make use of the announcement.

\section{Privacy}

Privacy is an issue that occurs when a user wants to explicitly restrict access to some groups of contacts for some types of status message or even only for a particular status message. It is usually related to groups of higher granularity, like the case of separating status messages for work and private contacts. People usually perceive some content types (like feelings and moods or travel experiences) to be suitable only for closer contacts or contacts of a more private nature, while those status messages should be kept private from some other (more professional) groups of contacts.

Some users, on the other hand express concern about the possibilities to track their status messages to the past and draw conclusions about their personality which would be out of their control. The concern is expressed about the uncontrolled data integration possibilities across services and attempts to integrate status messages with other content about the user and thus perform some spy-like behavior.

\section{The PRESENCE DiAMOND}

Once we acknowledge that many status messages have an intended audience and that access to them should in some cases be restricted to that particular audience (in cases of confidential messages), it becomes clear that one user might have different status messages for different audiences at the 
same time.

In fact, emitting different information (appearances) to different groups of observers is not restricted to status messages, but spans the whole notion of online presence. By the term online presence we refer to the totality of information that allows perceiving one's presence in online communities. Apart from status messages as an element of presence, availability for interaction might also have a faceted nature and be different for different groups at different times. One can easily imagine a working situation where a user is available for interaction only with his work colleagues and busy for all the others. Access to different presence information might also be given only to specific groups of contacts (like in the case of sharing the current location only with closest friends).

Therefore, there is a need to look at the notion of online presence as a faceted phenomenon. For this reason we introduce the notion of the presence diamond (Figure 1.) to capture the faceted nature of presence and the need to appear differently to different groups of people.
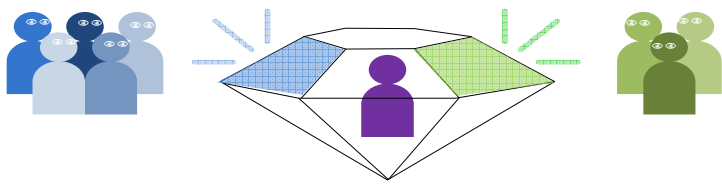

Figure. 1. The Presence Diamond ${ }^{2}$

The notion of presence diamond allows us to look at a person's online presence as a diamond whereby different observers are introduced to different facets of the diamond. Facets differ among themselves in different types of presence data that is accessible by observers of a facet (like in cases where one group of observers can access a person's location, availability and a status message, and another group can access only the status message), different granularity of data (like in the case of sharing the exact location with closest friends and only the current city/country with strangers), and in different data that is emitted to different observers (like having different status messages and different availability for different groups of contacts).

Even though we focus on status messages in this paper, we will look at the problem of directing status messages to their intended audience as a sub-problem of enabling faceted online presence, and will therefore favor solutions general enough to address the faceted nature of presence as a whole.

\section{INCOMPLETE WAYS TO DEAL WITH StATUS MESSAGE DIRECTING}

Some ways to direct status message updates to a particular audience already exist. In this section we present the workarounds found and applied by users, as well as solutions

\footnotetext{
${ }^{2}$ The figure and the notion of the Presence Diamond are strongly inspired by the notion of the diamond of digital identity, that Mike Roch, Director of IT Services at University of Reading, introduced at the Eduserv Digital Identity Workshop in London, January 08, 2009
}

developed as features of Social Web sites. For each of these solutions we discuss its incompleteness.

\section{A. User Workarounds}

Some users manage to separate their contacts on different Social Web services, by taking into account the nature of relationship with a particular contact. For example, a number of users maintain a list of work-related contacts on twitter while having a more personal network of friends on Facebook, and then share different status message updates for the different audiences. This way status messages related to private life can be kept confidential from work colleagues, and personal friends don't have to be bothered by work related postings. However, the fact that some contacts use only one social network stands in the way of such a separation. If some of the user's work colleagues use only Facebook, then maintaining the separation would mean not connecting at all with those persons. Apart from this limitation, if the separation by purpose is not done at the start, it is hard to impose it once the user has accepted different types of contacts to his/her social network.

Another way to deal with the identified issues is just to restrict oneself to publishing only status messages acceptable for the wide audience. Some users choose not to publish too personal status messages because work-related contacts might see them, and not to publish work-related status messages because they might not be of interest to their friends. This approach limits the potential of status message sharing in a great deal excluding many professional and staying-in-touch use cases.

\section{B. Solutions developed by Social Web Sites}

Solutions for niche microblogging and micro-broadcasting began to emerge recently. Those Social Web sites allow for broadcasting of status messages in closed communities (like in ShoutEm ${ }^{3}$ ) or to people gathered around a certain interest (like in $\left.\mathrm{Static}^{4}\right)$. However they mostly require intended recipients of the status message updates to join each closed community which can get quite complicated having in mind the number of intended audiences a user might have. This approach certainly leads to social network fatigue - a phenomenon of loss of motivation to participate in yet another social network when confronted with joining many social networks and building identities on them.

The new service $\mathrm{E}^{5}$ can be used to manage adding different people to different social networks according to the nature of the acquaintance (e.g. adding friends to Facebook and business contacts to MySpace). However, it is hard to enforce this separation since not all users are present on each of those networks and therefore some of connections might be lost if they do not meet the purpose one user has given to his/her social network account.

\footnotetext{
${ }^{3}$ http://www.shoutem.com/

${ }_{5}^{4} \mathrm{http} / / / \mathrm{www} . \mathrm{static} \cdot \mathrm{com} /$

${ }^{5} \mathrm{http}: / / \mathrm{www} . \mathrm{mynameise} . \mathrm{com} /$
} 


\section{THE LiNKED DATA WAY}

The term Linked Data [2] refers to publishing and interlinking structured data on the $\mathrm{Web}$ in $\mathrm{RDF}^{6}$ with the assumption that the value and usefulness of data increases the more it is interlinked with other data. This effort to publish the data online using open standards and interlink data sources is aimed at transforming the Web of documents towards a more (re)usable, machine readable Web of Data.

We argue that additional semantics describing a status message, as well as semantics (partially already published as Linked Data) describing users and their current context can be helpful to direct a status message to its intended audience, and thus reduce information noise and contribute to ensuring privacy. In particular we argue that currently available Linked Data sources can help define the intended audiences of status messages, relying on user properties described in those sources (interests, locations, social graph, etc.)

To enable publishing and exchange of such additional semantics, we decided to enrich an existing vocabulary - the Online Presence Ontology (OPO) ${ }^{7}$ - with the information about intended audience of a status message. The Online Presence Ontology presented in [3] provides a way to describe a user's current state of presence in the online world, including his/her availability for interaction, current status message, location and other elements of context. As such this vocabulary can be elegantly complemented with a way to direct a status message (or even the whole notion of Online Presence) to a certain audience. To enable this, we have extended the OPO with the notion of Sharing Space.

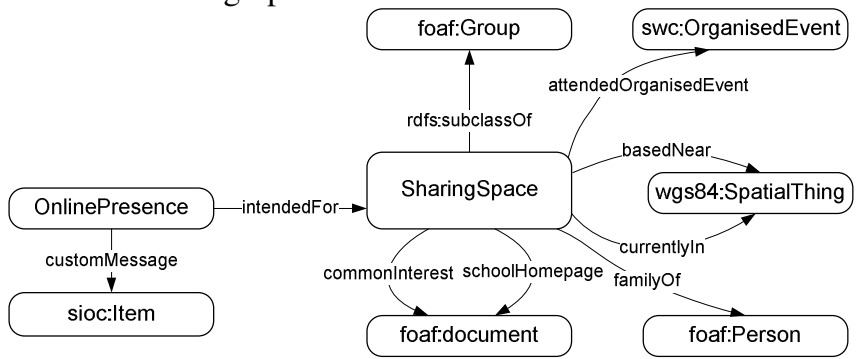

Figure 2. An excerpt from the Online Presence Ontology

A Sharing Space, in our specification, is a group of people (or agents) with whom particular information can be shared. As shown on Figure 2, the onlinePresence, encompassing (among other properties) the current status message of a user, can be connected to its intended audience through a property intendedFor by linking it with the notion of sharingspace. The status message itself is represented using the Item concept from the $\operatorname{SIOC}^{8}$ [4] ontology in order to enable replies to the status message and make use of this concept's suitable semantics. Sharing Space is also enriched with a list of properties to allow representing of the common attributes that bound members of the Sharing Space together (e.g., common interest, common current location). In order to express the

\footnotetext{
${ }^{6}$ Resource Description Framework http://www.w3.org/RDF/

${ }^{7} \mathrm{http}: / /$ www.milanstankovic.org/opo/

${ }^{8} \mathrm{http}: / /$ sioc-project.org/
}

semantics of those attributes we relied on concepts from

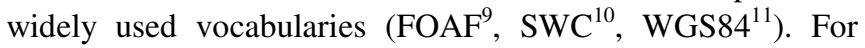
more details about the ontology design we refer the readers to the project website and the ontology specification ${ }^{12}$.

By identifying people who are intended to receive a status message, the notion of Sharing Space can help software systems to deliver status messages to specific people (members of the Sharing Space) and thus deal with information noise and even ensure confidential status message exchange.

In order to properly define Sharing Spaces according to the needs of real life scenarios, we will rely on the results of our user study, presented in Section 2. According to our study results, some of the major ways to define the intended audience are: friends of a certain friend; people having a certain interest; friends from a particular online community; people being in a certain location; people having a certain nature of relationship with the user; people who were affiliated in the same institution; and custom assembled groups of contacts.

A lot of information needed to define those groups (users' current and permanent locations, interests, friends' lists, etc.) is already available on the Social Web, and many sources already publish this data using vocabularies such as FOAF and SIOC [4]. Relying on those existing resources, Sharing Spaces could be dynamically defined using simple SPARQL ${ }^{13}$ queries that could identify the members of a particular Sharing Space by collecting data across different data sources. We believe that this way of defining Sharing Spaces is flexible enough to cover the needs of real life scenarios identified in our user study, and we will illustrate it on an example in the following subsection.

When proposing to use data from various distributed datasets, we should acknowledge that executing queries over distributed datasets might be a challenging task. However, this challenge has already attracted researchers to develop solutions for this distributed scenario. One of them is a system DARQ [5], an engine for federated SPARQL queries.

Apart from specifying Sharing Space members using SPARQL, the new version of the OWL language ${ }^{14}$, currently available as OWL 2 Working Draft [6] will provide a way to define Sharing Spaces through richer restriction axioms such as property chains. Property chains would allow to state that if a user satisfies a certain property then he is automatically a member of a Sharing Space. We also believe that the emerging Rule Interchange Format [7] (currently a working draft) will be a useful way to define and exchange Sharing Space definition rules across different systems that may use different rule languages internally.

\footnotetext{
${ }^{9}$ Friend-f-a-Friend vocabulary http://xmlns.com/foaf/spec/

${ }^{10}$ Semantic Web Conference Ontology

http://data.semanticweb.org/ns/swc/swc_2009-05-09.html

${ }^{11}$ World Geodetic System ontology http://www.w3.org/2003/01/geo/

${ }^{12}$ Other properties and classes introduced to support the notion of Sharing

Space can be found in the specification document

http://www.milanstankovic.org/opo/specs/

${ }^{13} \mathrm{http} / / / \mathrm{www}$.w3.org/TR/rdf-sparql-query/

${ }^{14}$ http://www.w3.org/2004/OWL/
} 


\section{A. Scenario of Use}

To better illustrate the flexibility of our approach and the usefulness of Linked Data, we present a scenario of publishing a status message together with a dedication to a particular Sharing Space. Figure 3 will serve as a graphical support to our explanations.

In this scenario, our example user Harry is organizing a reunion for his friends from the Semantic Web community. The reunion will take place in Paris, and Harry wants to announce it in his status message.

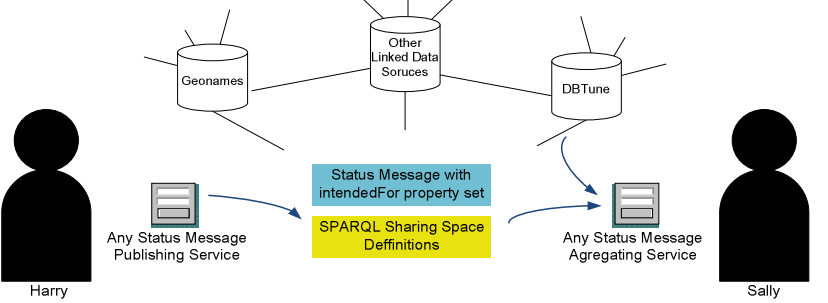

Figure 3. Publishing a Status Message

Thanks to the open nature of Semantic Web technologies, any status message publishing service (including microblogging platforms, social networks, chat platforms) can publish a status message and describe it using the OPO vocabulary. So, Harry's status message publishing service can make the semantically described message available to all status message consuming services. It can further associate it with a particular audience, by using the intendedFor property and the concept of a sharingspace. Along with OPO data about the status message itself, Harry's service can publish a SPARQL query to define the members of the Sharing Space. In our case, since Harry's message is intended for people interested in Semantic Web who are currently in Paris, the SPARQL Query would look like shown on Figure 4.

To make better use of the data available in Linked Data sources, we can reuse existing URIs used by those sources. In our example we rely on the Geonames ${ }^{15}$ URI for Paris, to uniquely identify this geographical location.

Once the message is available together with its semantic description, and a Sharing Space definition, other services can consume it and make it available to their users. Let us take another example user, Sally. She is Harry's friend, interested in Semantic Web and currently visiting Paris (according to her last published status message with associated geographic location information). Although Sally is not using the same status message publishing service as Harry, her Social Network (SN) service, can retrieve semantically described status messages and SPARQL queries defining Sharing Spaces. Since information about Sally's interest is available in one of her FOAF files, and available to her SN, and since her current location is also known to SN, applying the SPARQL query from Figure 4 will put Sally in SwPeopleInParis Sharing Space - the one Harry's status message is intended for.

Sally's interface for browsing status messages can now make sure that status messages intended for her get to her attention and somehow stand out from the abundance of other status messages put online by her friends and other people.

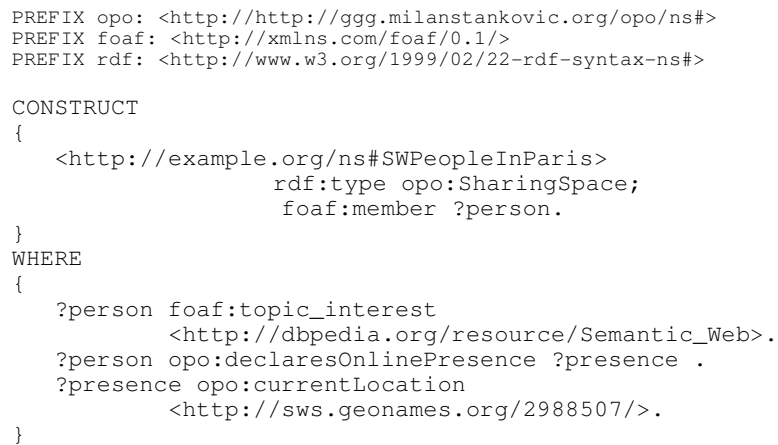

Figure 4. Example definition of a Sharing Space

\section{B. Some Benefits of Sharing Spaces}

Using the definitions of Sharing Spaces, like those shown in this paper, and publishing status messages that rely on the extended OPO vocabulary can help direct a status message to its audience. As opposed to solutions where particular (sometimes even closed) services are used to dedicate a status message to a certain group of people, our approach offers a way to dedicate a status message to a certain audience regardless of the service being used to publish them and present them. It is the use of widely accepted Semantic Web standards (e.g., $\mathrm{RDF}(\mathrm{S})$ and $\mathrm{OWL}$ ) that make the intended audience specifications universal and thus applicable everywhere.

The approach also allows to take into account the ever changing nature of user-related data, since membership in a Sharing Space can be defined through a property and not by naming particular members. Therefore users can belong to a sharing space at one time when they satisfy a certain condition (e.g. currently located in Paris), and not belong to it at all other times.

Apart from combating status message overload and helping relevant messages to reach their audience, Sharing Spaces can serve as a ground for ensuring privacy and confidential status message sharing. Our approach is based on the idea [8] that ensuring trust and privacy on the future Web can be grounded on the interlinked graph of data (i.e. Linked Data) and policies that take advantage of existing data sources. The introduced change in the OPO vocabulary is a first step in this direction, allowing to specify the intended audience of a status message by reusing existing (linked) data on the Web. Further mechanisms to enforce the delivery of a status message to the specified intended audience can be built on top of our presented solution. The advantage of this approach is that dedicating a status message to its audience is quite a general solution, addressing at the same time the challenge of dealing with information noise, and being the ground for ensuring the confidential status message sharing. 


\section{RELATED WORK}

Similar to our use of SPARQL to define sharing spaces i.e. intended audience groups, Alessandra Toninelli et al. [9] use RDF and SPARQL triple patterns to build social graph aware policies. Using triple patterns different policies can be created to grant access to user's attention (e.g., ring her phone). However this work is more related to mobile devices as it strongly reflects the specifics of communication using a mobile device, and in this sense it is complementary to our work in effort to make use of social data available in Linked Data sources to enhance user's interaction with devices and make her communications more adapted to her current situation. Another point of difference is that the socially-aware policy model is more concerned at granting/restricting access to a certain resource than dedicating/directing presence information to a certain audience.

\section{CONCLUSIONS AND FUTURE WORK}

In this paper we presented the results of our user study, based on qualitative research techniques, which was aimed at identifying the nature of problems surrounding status message publishing. Our study emphasized the need to direct a status message to a particular audience in order to deal with major issues like: Lack of Understanding, Significance, and Privacy.

We have shown how users try to deal with those issues and what solutions did the Social Web sites come up with to help with directing a status message to a certain audience. However, we judged all those solutions as incomplete either because they require users to join particular status sharing networks or because they restrain users from publishing certain types of status messages.

Our solution to the problem of dedicating a status message to a particular audience is based on providing semantic descriptions of intended audience and taking advantage of existing data about users published as Linked Data on the Web. Particularly we rely on a small extension of the Online Presence Ontology that allows for associating the intended audience information to a status message. Since the solution is based on Semantic Web technologies it allows a high level of interoperability and gives the intended audience information the ability to flow across different status message sharing services. Moreover, our semantic descriptions of intended audiences possess the ability to collect the intended audience members information from different Linked Data sources across the Web, which makes them universal.

Our future work will consist in evaluating the practical aspects of our proposal by extending the distributed microblogging platform $\mathrm{SMOB}^{16}$, described in [10] to publish and take into account the intended audience information through the use of new notion of Sharing Space introduced in the Online Presence Ontology. The new version of SMOB will make use of data available as Linked Data on the Web to create refined descriptions of audience for its status messages.

\footnotetext{
${ }^{16} \mathrm{http}: / /$ smob.sioc-project.org/
}

While the simple publishing and taking into account of intended audience information would be sufficient to combat the information noise problem, encompassing both issues of Lack of Understanding and Significance; some additional access control mechanisms must be employed to ensure that the intended audience specifications are properly applied across the Web. As a solution to access control we are considering to use the FOAF + SSL protocol [11] - a lightweight solution for authentication and authorization, based on the semantics exposed using the widespread FOAF vocabulary. The OpenID ${ }^{17}$ framework for providing a single digital identity across the internet can also elegantly contribute to achieve simple access control. OAuth ${ }^{18}$ authorization protocol could also be helpful in ensuring secure exchange of intended audience information across different services on the Social Web.

Although our solution for directing a status message to its audience is flexible in specifying the intended recipients of the status message, a lot of work remains to be done to ensure that the unintended recipients do not get access to it. We see the presented extension of OPO and the notion of Sharing Space as a first step in this direction.

\section{REFERENCES}

[1] Glaser, B. \& Strauss, A. Discovery of Grounded Theory. Chicago: Aldine, 1976

[2] Berners-Lee, T. Design Issues: Linked Data., 2006, http://www.w3.org/DesignIssues/LinkedData.html

[3] Stankovic, M. Modeling Online Presence. In: Proceedings of the First Social Data on the Web Workshop, Karlsruhe, Germany, 2008.

[4] Breslin, J.G., Harth, A., Bojars, U. \& Decker, S. "Towards Semantically-Interlinked Online Communities", Proceedings of the 2nd European Semantic Web Conference (ESWC '05), LNCS vol. 3532, pp. 500-514, Heraklion, Greece, 2005.

[5] Quilitz, B., Leser, U.: Querying Distributed RDF Data Sources with SPARQL. In: ESWC. (2008) Available: http://www.eswc2008.org/final-pdfs-for-web-site/qpII-2.pdf

[6] Motik, B., Patel-Schneider, P.F. \& Parsia, B. OWL 2 Web Ontology Language: Structural Specification and FunctionalStyle Syntax, 2008, http://www.w3.org/TR/2008/WD-owl2syntax-20081008/

[7] Boley, H., Hallmark, G., Kifer, M., Pasche, A., Pollares, A. \& Reynolds, D. RIF Core, 2008, http://www.w3.org/TR/rif-core/

[8] Passant, A., Kärger, P., Hausenblas, M., Olmedilla, D., Pollares, A. \& Decker, S. Enabling Trust and Privacy on the Social Web, In Proceedings of W3C Workshop on the Future of Social Networks, 15-16 January 2009, Barcelona,

[9] Toninelli, A., Khushraj, D., Lassila O., and Montanari, R.: Towards Socially Aware Mobile Phones. In: Proceedings of the First Social Data on the Web Workshop, Karlsruhe, Germany, October 27, 2008, CEUR Workshop Proceedings, ISSN 16130073 ,

[10] Passant, A., Hastrup, T., Bojars, U. \& Breslin, J. Microblogging: A Semantic Web and Distributed Approach. In: Proceedings of the 4th Workshop on Scripting for the Semantic Web, Tenerife, Spain, June 02, 2008, CEUR Workshop Proceedings, ISSN 1613-0073,

[11] Story, H. FOAF \& SSL: Creating a global decentralized authentication protocol, 2009. In W3C Workshop on the Future of Social Networking, 15-16 January 2009, Barcelona, Spain.

\footnotetext{
${ }^{17} \mathrm{http}: / /$ openid.net/

${ }^{18}$ http://oauth.net/
} 